\title{
Un análisis econométrico sobre la influencia de la apertura comercial, capital humano, inversión y trabajo en la desigualdad en el Perú (1997-2019)
}

\author{
Martha Sofía de la Cruz Flores \\ Universidad San Ignacio de Loyola (Lima, Perú) \\ sofiadelacruzflores@outlook.com \\ iD https://orcid.org/0000-0002-2790-9412
}

\author{
Mayra M. Camino Escudero \\ Universidad San Ignacio de Loyola (Lima, Perú) \\ mayracaminoescudero@gmail.com \\ (iD) https://orcid.org/0000-0001-9246-7828
}

\begin{abstract}
Resumen
El objetivo de este artículo se centra en analizar el impacto de la apertura comercial, el capital humano, el crédito privado y variables relacionados al trabajo y la especialización en la desigualdad de ingresos en el Perú durante el período de 1997 a 2019. Se emplea un modelo de regresión lineal múltiple basado en la metodología de mínimos cuadrados múltiples (MCO), usando datos de series de tiempo que matematizan la relación entre estas variables y la desigualdad de ingresos, que toma en consideración la especialización de la oferta laboral. Se comprobó la relación entre las variables independientes: capital humano y la apertura comercial, y la variable dependiente, el Gini, midiendo la desigualdad en el modelo final. Es importante mencionar que se han considerado los efectos de hechos históricos que afectaron al mercado en 2007, 2011 y 2019. El debate acerca del verdadero impacto de la apertura económica en la desigualdad de ingresos ha estado presente a lo largo de la historia. Por ello, la pertinencia de este trabajo radica en encontrar explicaciones prácticas en un escenario de profunda inestabilidad política y económica. Se busca hallar una de las causas de la desigualdad de ingresos que viene acompañando al Perú desde hace décadas. Dado que no se ha visto una reducción significativa, se requiere demostrar qué tipo de efecto tiene la globalización -durante las últimas dos décadas- en la lucha contra la desigualdad de ingresos en la sociedad peruana. Los resultados obtenidos demuestran que, en este caso, la apertura comercial sí tiene un impacto positivo en la reducción de la desigualdad de ingresos. Así mismo, se encuentran resultados inconclusos respecto de la naturaleza de la especialización y capacitación del capital humano en un entorno de apertura económica. En estos, se observan comportamientos contradictorios con la teoría económica y los estudios empíricos.
\end{abstract}

\section{Palabras clave \\ Desigualdad de ingresos, apertura económica, globalización, Gini}

\section{(c) (1)}




\title{
An econometric analysis of the influence of trade liberalization, human capital, investment, and work on inequality in Peru (1997-2019)
}

\author{
Martha Sofía de la Cruz Flores \\ Universidad San Ignacio de Loyola \\ mdelacruz@vidawasiperu.org \\ iD https://orcid.org/0000-0002-2790-9412
}

\section{Mayra Miluska Camino Escudero \\ Universidad San Ignacio de Loyola \\ mayracaminoescudero@gmail.com \\ ORCID pendiente}

\begin{abstract}
Resumen
The aim of this article focuses on analyzing the impact of trade liberalization, human capital, private credit, and variables related to work and specialization on income inequality in Peru during the period from 1997 to 2019. A multiple linear regression model based on the multiple least squares (OLS) methodology is applied, using time series data that mathematizes the relationship between these variables and income inequality, considering the specialization of the labor supply. The relation between the independent variables, human capital and trade openness, and the dependent variable, the Gini, was verified by measuring inequality in the final model. It is important to mention that the effects of historical events that affected the market in 2007, 2011 and 2019 have been considered. The debate about the true impact of economic openness on income inequality has been present throughout history. Therefore, the relevance of this work lies in finding practical explanations in a scenario of deep political and economic instability. We seek to find one of the causes of the income inequality that has been present in Peru for decades. Since a significant reduction has not been seen, it is necessary to demonstrate what type of effect globalization has -during the last two decadesin the fight against income inequality in Peruvian society. The results obtained show that, in this case, trade liberalization does have a positive impact on reducing income inequality. Likewise, inconclusive results are found regarding the nature of the specialization and training of human capital in an environment of economic openness. In these, contradictory behaviors are observed with economic theory and empirical studies.
\end{abstract}

\section{Palabras clave}

Income inequality, economic openness, globalization, Gini 


\section{Introducción}

En la literatura económica, se ha explorado desde diferentes ángulos la relación entre apertura económica, crecimiento, pobreza y desigualdad debido a la importancia que estos puntos tienen para determinar la calidad de vida de las personas. Pese a los grandes avances que se ha podido observar en la reducción de la pobreza, la desigualdad se ha incrementado exponencialmente o no se ha reducido con suficiente rapidez en determinados países pese al crecimiento económico, que ha sido impulsado en gran medida por la apertura económica (International Monetary Fund [IMF], 2015). Por ello, la presente investigación tiene como objetivo analizar el impacto de la apertura económica, capital humano, crédito al sector privado, trabajo en el sector agrícola y comercial en la desigualdad del Perú durante el período de 1997 a 2019.

Desde finales de la década de 1980, las políticas económicas se han enfocado en el impulso del crecimiento y liberación económicos. Diferentes posturas han indicado que esta liberación es la responsable de una distribución más equitativa del ingreso o la que ha desencadenado la desigualdad (Morán, 2015). Autores como Kaldor (1957), Kuznets (1955) y Forbes (2000) afirman que existe una relación positiva entre crecimiento y desigualdad, pero que esta es compensada por la eficiencia de sectores más ricos. La visión clásica del comercio internacional peca de centrarse en el largo plazo, sin considerar las consecuencias de la transición o de la distribución de ingresos a favor del crecimiento. Por este motivo, autores modernos -como Piketty (2013) - realizan una crítica al sistema y afirman que los países de mayor crecimiento (China o Corea) se han centrado en fortalecer a su población y no solo a abrirse internacionalmente para crecer. Ello se evidencia de forma más clara debido a los aportes realizados por Morán (2015), y Germán y Escobedo (2009), quienes sostienen que la apertura solo trae desigualdad y esta no es contrarrestada por el mercado, por lo cual es necesaria la intervención del Estado.

Pese a los avances en algunos de sus países, América Latina sigue siendo considerada la región más desigual del mundo. A pesar de la tendencia decreciente que expresa el coeficiente de Gini en los últimos años, las correcciones metodológicas pueden visibilizar la desproporcionalidad de ingresos del $1 \%$ más rico a comparación de casi toda la población (Comisión Económica para América Latina y el Caribe [Cepal], 2019). La preocupación por la desigualdad latinoamericana no solo proviene de la percepción de ingresos, sino de problemas estructurales relacionados al género, la etnia, clase, edad, etc. (Cepal, 2016). El Perú no es ajeno a esta tendencia. Ha reducido su desigualdad general de forma progresiva, pero, si se analiza con mayor profundidad, se puede observar que dentro del país hay regiones que se han vuelto aún más desiguales (Castillo, 2019).

Para la determinación del impacto de la apertura comercial, el capital humano, el crédito al sector privado y variables relacionados al trabajo y la especialización en la desigualdad de ingresos, se utilizó el modelo econométrico Mínimos Cuadrados Ordinarios de series de tiempo, basado en la propuesta metodológica planteada por el Fondo Monetario Internacional (2007), en el cual se establece la clásica relación entre crecimiento y desigualdad, y se adicionan variables relacionadas a la apertura comercial, 
el capital humano, la inversión y el trabajo en diferentes sectores. Con ello, se pretende medir el impacto de las dos variables principales y, adicionalmente, observar cómo otros factores económicos han influido en estas. Por otro lado, cabe resaltar que los datos utilizados para la realización del estudio han sido recopilados del Banco Mundial y Penn Datatable, como fuentes internacionales, y el Banco Central de Reserva del Perú, como fuente local.

La presente investigación se encuentra distribuida de la siguiente forma: en primer lugar, se iniciará con la descripción del problema. En segundo lugar, se realizará una exposición de la información recopilada a lo largo de la historia económica por diversos autores, contraponiendo teorías clásicas de comercio con las críticas actuales. En tercer lugar, se realizará la exposición del modelo y los datos para realizar el análisis cuantitativo. Luego, se explicarán los resultados obtenidos por el modelo. Al finalizar, se presentarán las conclusiones y recomendaciones del caso.

\section{Problema de investigación}

En las últimas décadas, se ha vuelto evidente que el mundo es mucho más rico: el incremento de la riqueza mundial en las últimas décadas ha sido exponencial, y creció $66 \%$ en el período de 1995 a 2014. No obstante, ello no significa que ese incremento en la riqueza se haya dado de manera proporcional para todos. Existen desequilibrios estructurales ocultos tras la fachada de crecimiento. El Perú, entre 2002 y 2012, fue uno de los países con crecimiento acelerado en toda la región, lo que le permitió reducir la pobreza e incrementar los empleos, y las exportaciones fueron uno de los principales motores para mantener a flote la economía (Banco Mundial, 2020). El estado de esta última no es solo el crecimiento; de hecho, pese a que la pobreza ha disminuido considerablemente, de $20 \%$ en 2006 a $12.7 \%$ en 2016, la desigualdad, medida por el coeficiente de Gini, se ha mantenido prácticamente igual (INEI, 2017).

Las implicancias de la desigualdad de ingresos no solo están relacionadas al dinero percibido por las personas, sino que son interseccionales al acceso a la salud, beneficios sociales, trabajo, educación, servicios básicos, entre muchos otros (United Nations, s.f.). Las Naciones Unidas (s.f.) expresan su preocupación por la desigualdad y sus efectos económicos y no económicos, y consideran necesario un cambio en el accionar, en el que los países, las empresas y las personas dirijan sus esfuerzos por alcanzar los objetivos de desarrollo sostenible con el fin de construir un mundo más equitativo, justo y sostenible.

Dentro de este marco, se puede plantear la siguiente pregunta de investigación: ¿Cuál ha sido el impacto de la apertura económica en la desigualdad de ingresos en el Perú en el período de 1997 a 2019? Para brindar una respuesta, se empleará un modelo que integre ambas variables: la apertura comercial medida por las exportaciones e importaciones como porcentaje del PBI y la desigualdad de ingresos determinada por el Gini, así como el capital humano, para poder medir la influencia de esta variable en la reducción de desigualdades. 


\section{Justificación de la investigación}

Las Naciones Unidas (s.f.) alertan de las consecuencias de la desigualdad en la calidad de vida de las personas: no solo se trata de las afecciones al factor económico sino al acceso a servicios de primera necesidad, salud, educación, saneamiento, entre otros que garantizan una vida digna. Bajo esta premisa, es sumamente preocupante caer en cuenta de que el mundo es cada vez más rico; sin embargo, al mismo tiempo, la distribución de esa riqueza se produce de forma arbitraria y se intensifican las desigualdades, y así se dan situaciones en las cuales solo el 50 \% de la población mundial tiene acceso a la protección social (United Nations, 2019). La situación se agrava aún más en América Latina, que es considerada la región más desigual del mundo, donde una mujer chilena nacida en un barrio pobre de la capital tiene 18 ańos menos de esperanza de vida que otra mujer nacida en un barrio rico (BBC, 2020).

El crecimiento económico sostenido que se ha experimentado en todo el mundo -especialmente en muchos países de Latinoamérica desde fines de la década de 1980- se ha debido principalmente al énfasis en políticas que buscan la facilitación del comercio internacional y la erradicación de las trabas que impedían la apertura (Morán, 2015). Aquello se ha dado con base en las teorías clásicas y neoclásicas del comercio internacional que enfatizan la apertura para potenciar el crecimiento a corto, mediano y largo plazo. Con ello, se ha logrado disminuir drásticamente la pobreza, pero se ha incrementado o se ha mantenido constante la desigualdad (IMF, 2015).

Con Ricardo, Smith y otros autores clásicos, se evidencia la importancia de la especialización para la obtención de la ventaja competitiva que traiga consigo el crecimiento económico desde el comercio internacional (Oros, 2015). Así mismo, Oros (2015), en su análisis del modelo de HOS, explica cómo este se basa en el supuesto de que, en el comercio entre los países, el propietario de la industria más competitiva será el ganador y mejorará las rentas de su sector, pero que los otros sectores no ganarán. Autores como Kaldor (1957) consideran que, si un país desea crecer, la desigualdad será condición necesaria para lograr ese objetivo, debido a que el crecimiento se logra enfocando las políticas económicas favorecedoras en los sectores más productivos (los más ricos y ahorradores) y teniendo como perdedores a los sectores menos productivos.

La visión clásica no considera la importancia de los sectores perdedores; solo considera que lo obtenido por el sector ganador compensará la desigualdad general. Con esto, se puede visibilizar la dualidad del libre comercio: crecer o igualar las condiciones. Por este motivo, la presente investigación tiene como objetivo analizar el impacto de la apertura comercial en la desigualdad, considerando la influencia que han tenido otros factores en esta. Por otro lado, aportará mayor evidencia empírica del funcionamiento de las políticas comerciales actuales y de la marginalización que están viviendo los sectores menos beneficiados de la economía, y permitirá a las autoridades pertinentes -el Ministerio de Economía y Finanzas, el Ministerio de Desarrollo e Inclusión Social, y el Ministerio de Comercio Exterior y Turismo- tener más herramientas para llevar mejor las políticas comerciales promulgadas. Así mismo, se busca visibilizar un problema estructural en la economía peruana y adquirir mayores herramientas de análisis en la lucha por la reducción de la desigualdad con el fin de tener un país más equitativo, justo y sostenible. 


\section{Estado del arte y marco teórico}

Hoyos y Lusting (2009) explican que, pese a la diversidad de enfoques metodológicos que existen, no se ha llegado a un acuerdo con respecto a la relación entre la apertura comercial, y la desigualdad de ingresos y la pobreza. Los resultados, dependiendo del estudio y la metodología, variarán; los precios pueden reducirse; los bienes pueden incrementar; las industrias pueden verse perjudicadas o ganar. El efecto positivo o negativo dependerá del caso por analizar. En el caso ecuatoriano, Morán (2014) afirma que la desigualdad de ingresos y la apertura comercial son directamente proporcionales. Es decir, al abrirse económicamente un país, la desigualdad incrementará a lo largo del tiempo, aunque esta exposición al mercado internacional traerá consigo crecimiento económico. En su estudio de México, Germán y Escobedo (2009) explican que, al inicio, la apertura sí trajo una disminución de la desigualdad, pero que esta no pudo mantenerse y se empezó a generar una divergencia, desigualdad, más marcada en los ingresos de las personas del país. Majeed (2010), en su análisis del caso asiático, concluye que la apertura comercial ha traído un incremento de la desigualdad de ingresos, debido, principalmente, a la acumulación de capital y el crecimiento económico. En otras palabras, la apertura activa una economía y hace que esta crezca, pero el beneficio no es homogéneo para todas las personas, con lo que se produce una disparidad en los ingresos.

Ezcurra y Pose (2014) analizan 22 países en desarrollo, y concluyen que la apertura comercial genera ganadores y perdedores. En su estudio, se aprecia la heterogeneidad de los resultados: unas regiones, países y personas ganan, mientras otros se ven perjudicados. En México, Grañudo (2014) expone que la apertura agrava la desigualdad incluso entre regiones de un mismo país, debido a que, al estar más cerca de Estados Unidos, el crecimiento de una ciudad o región se incrementa; esta afirmación es corroborada por Ismael (2007). De la misma manera, Castro y Aguilera (2016), en su análisis de México 1992-2014, concluyen que, en oposición a la teoría de Stolper-Samuelson, la apertura comercial no aportó positivamente en la reducción de la desigualdad de ingresos a largo plazo. Según el teorema de Stolper-Samuelson, los efectos de la apertura comercial varían de país en país dependiendo de su oferta laboral. Los países con mayor oferta de trabajo sin especialización sentirán un impacto más intenso que aquellos con nivel de especialización intermedia, que sentirán efectos menores o incluso negativos. Sin embargo, las regiones con mayor exposición a la liberación experimentaron una mejora en el salario que las menos expuestas.

Las investigaciones anteriormente descritas visibilizan una relación entre apertura comercial y desigualdad, pero no concluyen unánimemente si esta es positiva o negativa. Francke (2017), en su revisión histórica de las formas de estimación de la desigualdad económica en Perú, demuestra que todos los estudios tienen limitaciones dependiendo de los tipos de variables escogidas y las estimaciones que se realicen.

El siguiente modelo explica los efectos de la globalización en la desigualdad de ingresos, tomando en consideración puntos relevantes para las economías avanzadas y en desarrollo. Para el caso de las economías en desarrollo, se busca exponer la relación entre el desarrollo de los sectores agropecuarios e industriales con respecto a la desigualdad 
en un contexto de apertura, debido a la menor y mayor especialización de la mano de obra; así mismo, se analiza su relación con la inversión (crédito) y el efecto que tiene la especialización de la mano de obra. En este trabajo de investigación, se utilizará el modelo simplificado y adaptado del Fondo Monetario Internacional (2007):

$$
\begin{aligned}
& G I N I_{t}=B_{0}+B_{1} A P E R_{\mathrm{t}}+B_{2} C R E D_{\mathrm{t}}+B_{3} P O P S H_{\mathrm{t}}+B_{4} C A P H U M_{\mathrm{t}}+B_{5} A G R_{\mathrm{t}}+B_{6} I N D_{\mathrm{t}} \text { (1) } \\
& G I N I_{t}=\text { Coeficiente de GINI } \\
& A P E R_{\mathrm{t}}=\text { Apertura Económica (Suma de exportaciones importaciones \% PIB) } \\
& C R E D_{\mathrm{t}}=\text { Crédito al sector privado por depósitos en bancos y otras instituciones (\% PIB) } \\
& P O P S H_{\mathrm{t}}=\text { Población de } 15 \text { a más ańos con estudios secundarios o estudios superiores } \\
& C A P H U M_{\mathrm{t}}=\text { Índice del capital humano } \\
& A G R_{\mathrm{t}}=\text { Trabajo en el sector agrario } \\
& I N D_{\mathrm{t}}=\text { Trabajo en el sector industrial }
\end{aligned}
$$

Muhammad (2016) resume la relación de la tesis planteada por Perotti (1995), Forbes (2010) y Barro (1999), que relacionan el crecimiento, la desigualdad y la apertura comercial, reconociendo que, pese a las imperfecciones del mercado, la inversión en especialización del capital humano trae una reducción en la desigualdad de ingresos. Con ello, se comprueba la teoría del capital humano que afirma que, al incrementar el nivel de especialización y capacitación, las personas podrán aprovechar las oportunidades generadas por la apertura comercial y podrán aumentar sus ingresos. Sin embargo, Muhammad (2010) señala que una sociedad profundamente desigual que se abre al mundo comercial genera un escenario de inequidad en relación con las oportunidades que gozan las personas por su situación económica y social. Es decir, las personas podrán acceder a mayor o menor cantidad de oportunidades dependiendo de sus ingresos previos, lo cual mantiene la ventaja de unos sobre otros.

Según los estudios empíricos del FMI (2007), la apertura comercial ha demostrado ser positiva a la hora de incrementar los salarios de los sectores con menos capacitaciones e ingresos, debido al incremento de la actividad económica y la redistribución del mercado laboral, específicamente la reducción del trabajo en el sector agrícola. La distribución del empleo sufre un cambio radical a raíz de la apertura de posiciones laborales mejor remuneradas a las cuales solo es posible acceder mejorando el nivel de capacitación. Por eso, progresivamente, las fuerzas productivas migran a puestos con mayor nivel de especialización. Al contrario de esto, Castro y Aguilera (2016), para el caso mexicano, evidencian que, en relación con el capital humano, los trabajadores más calificados sufrieron de un deterioro en su salario real en comparación con aquellos menos calificados al generarse la apertura económica.

Wood (2002), basándose en teorías clásicas, sugiere que la desigualdad de ingresos disminuirá por la necesidad que se genera por la mano de obra no especializada en la apertura. Así mismo, el autor concluye que, pese a existir una reducción en la desigualdad de ingresos al producirse una apertura comercial, existen casos, como el latinoamericano 
(1980-1990) y el del este asiático (1960-1970), en los que no se cumple debido a los sesgos técnicos en contra de los trabajadores menos calificados. Por otro lado, Winters (2002) relaciona los impactos del comercio exterior, y plantea un modelo que centra su foco en el ingreso del hogar y relaciona las repercusiones que tienen los factores de producción y el consumo con referencia a la actividad agrícola. Sobre la base de su modelo, se puede ver el efecto de los aranceles con respecto a los ingresos reales de las personas:

$$
\frac{\partial Y}{\partial x}=\frac{\partial P_{k}}{\partial t}\left(Q_{k}^{s}-Q_{k}^{d}\right)
$$

Bajo esta relación, se entiende que el efecto inmediato de la apertura comercial será determinado por el cambio de los precios originados por la diferencia arancelaria. En este escenario, si los precios relativos bajan, los hogares percibirán un incremento en sus ingresos reales. En otros términos, el incremento de la cantidad de bienes que trae la apertura generará una reducción de los precios, lo que incrementará la capacidad adquisitiva de las personas. Cabe precisar que, en los sectores más pobres, el efecto de este cambio es escaso o nulo (Winters, 2002).

Salas et al., (2006) explican que la liberación económica latinoamericana producida en la década de 1980 generó efectos positivos relacionados al crecimiento económico y a asegurar la estabilidad macroeconómica en muchos países. No obstante, sectores como el agrícola sufrieron pérdidas claras con la apertura. El sector agrícola, al carecer de una reforma estructural y políticas coherentes, experimentó una pérdida de la productividad y no pudo aprovechar las oportunidades generadas por la liberación. López et al., (2020) determinan que, en el caso mexicano, la elasticidad de la demanda de trabajo de la industria manufacturera incrementó con la apertura comercial; es decir, se magnificaron las variaciones en el trabajo y las remuneraciones. Aquello origina que las condiciones laborales no puedan mejorar significativamente debido a la sensibilidad hacia los costos laborales, además de una mayor incertidumbre, inestabilidad en el mercado y menor posibilidad de sindicalización para exigir cambios.

Así mismo, cuando se analizan el crédito, apertura y la desigualdad, se puede observar una distorsión. Cuando una economía se abre al comercio exterior, pero tiene profundos problemas de desigualdad, esta tenderá a perpetuarse, ya que las personas que han nacido con más ingresos y en un mejor ambiente tendrán más ventajas que aquellas de sectores humildes (Muhammad, 2010). Por otro lado, según los estudios empíricos del FMI (2007), la apertura ha demostrado ser positiva para incrementar los ingresos de los sectores con menos calificaciones y menores ingresos, lo que se relaciona principalmente con el incremento de la exportación y la disminución del trabajo agrícola, que representa la productividad del sector.

Gómez et al., (2019) sostienen que, dependiendo del enfoque que se le dé al análisis del desarrollo del sistema financiero -medido por el crédito del sector privado en depósitos en bancos y otras instituciones financieras-, se pueden observar dos comportamientos: el desarrollo del sistema financiero incrementará las desigualdades o las disminuirá. Por un lado, el incremento del crédito y de la IED está relacionado al acceso financiero que 
el sector con más ingresos tiene; ello genera una desigualdad que se verá reflejada en la diferencia de ingresos (FMI, 2007). Desde una visión histórica, por otro lado, existe mayor evidencia para apostar por la reducción de la desigualdad, por lo que los autores consideran que es importante que las políticas públicas fomenten la inclusión financiera, y se priorice el crédito en educación e innovación (Gómez et al., 2019).

\section{Objetivos}

El análisis de la literatura con respecto al tema, como teorías y antecedentes pertinentes, permitieron determinar el enfoque del presente trabajo de investigación. Por ello, el objetivo general es analizar el impacto de la apertura económica en la desigualdad de ingresos en el Perú en el período de 1997 a 2019. Dentro del marco y lapso temporal analizado, también se busca determinar la influencia del desarrollo del empleo en los sectores agrícolas e industriales en la desigualdad de ingresos, describir la relación entre el desarrollo financiero y la desigualdad de ingresos, así como también describir la relación entre esta última y el capital humano.

\section{Método}

La presente investigación se desarrolla en un nivel explicativo (Esteban, s.f.), que se refiere al impacto de la apertura comercial, el capital humano, crédito al sector privado, el trabajo en el sector agrícola e industrial, y la capacitación sobre la desigualdad. Posee un diseño no experimental, ya que no se hace ningún tipo de variación a la muestra (Hernández et al., 2000). Es longitudinal o evolutivo con tendencia debido al análisis temporal en las variables de una determinada población. Por otro lado, es correlacional descriptivo dado que describe la relación que se manifiesta de forma orgánica entre las variables seleccionadas (Sousa et al., 2007).

El presente trabajo abarca una línea temporal que inicia en 1997 y termina en 2019, y consta de 22 observaciones. Se decidió tomar en consideración este período debido a que, antes de 1997 (década de 1980 e inicios de los ańos noventa) era irrefutable la crisis en la cual estaba sumergido el país. Para inicios de la década de 1990, se implementaron nuevas medidas de política económica y monetaria, lo que marcó un punto de inflexión desde 1997. Así se obtuvo tasas de crecimiento del PBI de hasta un $9.8 \%$ para 2008 (Del Álamo, 2011), y 2019 fue tomado en consideración debido a que fue el último año en que se presentó un crecimiento continuo y estable de la economía peruana.

Por otro lado, tanto las observaciones de la variable dependiente (índice de Gini) y las variables independientes (apertura comercial, crédito privado, popsh, capital humano, empleo en agricultura y empleo en industria) han sido recolectadas de la base de datos abierta del Banco Mundial y Penn World Data Table. 
Tabla 1. Variables

\begin{tabular}{|c|c|c|c|c|c|}
\hline Variable & Nombre & Unidad de medida & Tipo & Frecuencia & Fuente \\
\hline GINI & Índice de GINI & Unidades & Independiente & Anual & Penn Data Table \\
\hline APER & Apertura comercial (\% PIB) & Porcentaje & Independiente & Anual & Banco Mundial \\
\hline CRED & $\begin{array}{l}\text { Crédito al sector privado por } \\
\text { depósitos en bancos y otras } \\
\text { instituciones ( } \% \text { PIB) }\end{array}$ & Porcentaje & Independiente & Anual & Banco Mundial \\
\hline POPSH & $\begin{array}{l}\text { Población de } 15 \text { a más años } \\
\text { con estudios secundarios o } \\
\text { estudios superiores }\end{array}$ & Porcentaje & Independiente & Anual & Banco Mundial \\
\hline CAPHUM & Índice de Capital Humano & Unidades & Independiente & Anual & Penn Data Table \\
\hline AGRIC & $\begin{array}{l}\text { Trabajo en agricultura (\% total } \\
\text { del empleo) }\end{array}$ & Porcentaje & Independiente & Anual & Banco Mundial \\
\hline IND & $\begin{array}{l}\text { Trabajo en industria (\% total } \\
\text { del empleo) }\end{array}$ & Porcentaje & Independiente & Anual & Banco Mundial \\
\hline
\end{tabular}

Nota: Esta tabla muestra las variables con sus respectivas descripciones, unidades de medida, tipos, frecuencias y fuentes con el fin de resumir y simplificar la lectura.

\section{Resultados}

El desarrollo de este modelo econométrico está basado en un modelo de regresión lineal múltiple; de la misma manera, el análisis realizado para poder corroborar la pertinencia de este modelo en la teoría económica se llevó a cabo bajo las premisas de Gujarati \& Porter (2009).

Luego de comprobar la validez individual de cada variable, se pasa a realizar la prueba de causalidad de Granger, que demuestra la relación de causa entre una serie de tiempo y otra, en este caso, entre las variables regresoras y el regresando. De acuerdo con el análisis de causalidad de las series, se pudo determinar que existe bidireccionalidad entre al LGINI $\leftrightarrow \triangle L A P E R, L G I N I \leftrightarrow \triangle L P O P S H, L G I N I \leftrightarrow \triangle L C A P H U M, L G I N I \leftrightarrow \triangle L A G R, L G I N I \leftrightarrow$ $\triangle L I N D 10 \%$; por otro lado, existe unidireccionalidad entre $\triangle L C R E D \leftrightarrow L G I N I$ al $10 \%$.

El modelo planteado por la prueba de causalidad de Granger sugiere que

$$
L G I N I=f(\triangle L A P E R, \triangle L C R E D, \triangle L C A P H U M, \triangle L A G R, \triangle L I N D)(3)
$$

Al momento de estimar el modelo, los resultados indicaron que tiene un nivel de ajuste muy bajo, siendo el . Así mismo, el modelo demostró ser poco significativo globalmente, con un, y variables independientes no significativas, excepto por la variable de capital humano. Por el lado del cumplimiento de los supuestos básicos de la estimación por $\mathrm{MCO}$, se tuvo como resultado que estos cumplen las condiciones de homocedasticidad, normalidad, estacionariedad y linealidad, pero que presentan autocorrelación de residuales, por lo que se determina que este modelo no es pertinente para explicar la relación entre las variables explicativas y la variable explicada.

Ya que el modelo anterior presentaba problemas, se estimó un nuevo modelo. Este contará solo con dos variables independientes: debido a que las otras tres variables generaban poca significancia, así como problemas de autocorrelación. Así mismo, se 
incluyeron dummies aditivas y dicótomas para poder generar mayor fiabilidad en el modelo. Esto se debe principalmente al punto de quiebre observado en 2011 para capital humano y los picos que se vieron en 2009, 2011 y 2019 para la apertura comercial.

Tabla 2. $M C O$ corregido

\begin{tabular}{|c|c|}
\hline Variable & Coeficiente \\
\hline$C$ & $3.919244^{* * *}$ \\
\hline$\triangle L A P E R$ & $-0.303810^{*}$ \\
\hline$\triangle L C A P H U M$ & $3.369798^{* *}$ \\
\hline$D 2011 \triangle L C A P H U M$ & $-27.74695^{* * *}$ \\
\hline$D 2009$ & $-0.104875^{*}$ \\
\hline$D 2011$ & -0.08851 \\
\hline$D 2019$ & -0.051139 \\
\hline \multicolumn{2}{|c}{ Nota: ${ }^{*} \mathrm{p}<0.10,{ }^{* *} \mathrm{p}<0.05,{ }^{* * *} \mathrm{p}<0.01$} \\
\hline
\end{tabular}

Fuente: Elaboración propia

$$
\begin{gathered}
\text { LGINI }=3.919244-0.303810 * \triangle L A P E R+3.369798 * \Delta L C A P H U M-27.74695 * \\
D 2011 \triangle L C A P H U M-0.104875 * D 2009-0.08851 * D 2011-0.051139 * D 2019+\hat{\varepsilon}_{t}
\end{gathered}
$$

En este modelo, se puede ver que el $\mathrm{R}$ cuadrado ajustado se ha incrementado a un 0.879523 , lo que demuestra un muy buen ajuste del modelo. Presenta una buena significancia global, dado que el $F$ - estadístico $=0.0000$. Por el lado del cumplimiento de los supuestos de un MCO, se tiene un resultado positivo, y se llega a comprobar todos los supuestos del modelo, lo que demuestra un modelo eficiente y que explica a un alto nivel de confianza la relación entre la apertura comercial, el capital humano y la desigualdad económica, tomando en cuenta los efectos de los eventos históricos de 2009, 2011 y 2019.

\section{Discusión}

De acuerdo con los resultados, se pudo observar que el coeficiente de la apertura comercial es negativo (-0.303810), por lo cual, se rechaza la hipótesis nula. Pese a que existe una discusión constante de si la apertura incrementa o disminuye la desigualdad de ingresos de un país, en el caso peruano de 1997 a 2019, se puede afirmar que esta sí fue positiva para la disminución de la desigualdad. Por otro lado, los aportes de Winters (2002) sugieren que esto se debe, inicialmente, al incremento de la capacidad de compra de los consumidores internos a raíz de la disminución de los precios relativos. Así mismo, el autor señala que, al ser este una consecuencia de la apertura, cualquier cambio que surja en políticas comerciales afectará principalmente a las personas más pobres. Aquello está cimentado bajo las propuestas iniciales de Kuznets (1955), que afirmaba que el crecimiento, traído por la apertura, disminuiría progresivamente los índices de desigualdad. 
Con estos resultados, se puede concluir que la apertura, fomentada aún más por la globalización, continuará disminuyendo en el tiempo. Además, se pudo corroborar que la apertura comercial sí tiene efectos positivos en la reducción de la desigualdad. Esto puede estar ligado a la disminución de precios y el incremento del poder adquisitivo que se origina al abrirse un mercado y dejar pasar importaciones a precios más baratos. De la misma manera, se puede corroborar lo planteado por Lopez, Alavargonzález y Pérez (2006) al hacer la extensión de la hipótesis de Kuznets, que afirmaba que, a largo plazo, se tendría una disminución progresiva de la desigualdad debido al crecimiento empujado por el comercio exterior.

Cabe resaltar que los picos en el comercio exterior en 2009, 2011 y 2019 provocaron distorsión en el análisis a largo plazo de la variable de apertura comercial. El primero se debe principalmente a la crisis financiera que en el Perú fue sentida como una crisis de exportaciones; el segundo, al crecimiento récord de las exportaciones; y el tercero, a la disminución de las exportaciones tradicionales con respecto al año anterior.

Por otro lado, en el modelo final, se observa que el coeficiente del capital humano es positivo (3.369798), por lo que no se puede rechazar la hipótesis nula. El capital humano tiene una relación directamente proporcional a la desigualdad, así que, si una incrementa, la otra también lo hará. Esto contradice la teoría del capital humano, que planteaba que la inversión en cualquier tipo de educación incrementará los salarios, lo que terminará repercutiendo en la reducción de la desigualdad de ingresos. También contradice los estudios empíricos del FMI (2007), que seńalaban que se generaría una la movilización de la mano de obra a sectores de mayor calificación y remuneración con la apertura. Por otro lado, un análisis del teorema de Stolper-Samuelson da mayor claridad al sostener que la apertura del comercio se comporta de manera diversa en países con diferentes grados de especialización, y puede ser positiva en los de muy baja especialización o negativa cuando existe muy alta especialización. Aquello apoya las evidencias de Castro y Aguilera (2016) para el caso mexicano, en el que los trabajadores más calificados sufrieron un deterioro en su salario real al generarse la apertura económica.

Según Wood (2002), la brecha entre ingresos también se puede ver reducida debido a los cambios en los salarios que perciben las personas menos aventajadas económicamente y que tienen menos especialización al realizarse la apertura. En el análisis del StolperSamuelson, se entiende que los países vivirán de forma diferente su apertura comercial, puesto que los efectos que se tiene en países con mano de obra más calificada variarán a comparación de aquellos que no tengan capacitaciones. Los países con mayor especialización tendrán un menor efecto positivo de la apertura o incluso negativo. Aquello puede responder a por qué existe esta relación directamente proporcional entre el índice de Gini y el índice de capital humano. Pese a ello, es importante resaltar que la dummy multiplicativa del capital humano es negativa, lo que generaría discordancia con lo anteriormente expresado.

Debe resaltarse que la dummy multiplicativa del capital humano, que refleja el quiebre de la tendencia en los datos anuales a partir de 2011, es negativa, lo cual puede significar que, a partir de ese año, esta variable tenga efectos positivos en la reducción de la desigualdad de ingresos. 


\section{Conclusiones}

El actual contexto político y económico ha hecho necesario que se evalúen los ejes principales de la economía, y se busca evidencia empírica de si las recetas utilizadas para el crecimiento están teniendo un efecto positivo en la mejora de la calidad de vida de las personas. Durante el trabajo de investigación, se han enumerado las consecuencias atroces que trae la desigualdad a los seres humanos, agravando la pobreza y el acceso a servicios básicos. En este estudio, se ha analizado empíricamente la relación entre la apertura económica y los efectos que ha tenido la globalización de los sectores agrícola e industrial, así como el desarrollo financiero y del capital humano en la desigualdad de ingresos.

Así, se ha podido concluir que la apertura comercial ha tenido un efecto positivo en la reducción de la desigualdad de los ingresos a lo largo del tiempo, lo que puede ser atribuido a la mejora del poder adquisitivo que traen las exportaciones, o al incremento de trabajo para mano de obra poco o medianamente calificada. Por ende, se puede afirmar que, al incrementar en $1 \%$ la primera diferencia de la apertura comercial, el coeficiente de Gini disminuye en $-0.303810 \%$. Es importante mencionar que los picos vistos en el comercio exterior en 2009, 2011 y 2019 tuvieron efectos positivos y negativos con respecto a la desigualdad, y fueron esos años de decrecimiento o incremento en las exportaciones.

El capital humano ha tenido un efecto negativo en la reducción de la desigualdad de ingresos, lo que contradice la literatura de la teoría del capital humano, pero podría relacionarse con la diversidad de comportamientos que genera el comercio exterior en mano de obra calificada y no calificada, dependiendo del país con respecto a la desigualdad. Los resultados mostraron que un incremento del $1 \%$ en la primera diferencia del capital humano, incrementa, a su vez, en 3.369798 \% el coeficiente de Gini. Pese a ello, la dummy multiplicativa de dicha variable sí tiene un efecto positivo en la reducción de la desigualdad, lo que contradeciría al anterior resultado. Por ello, se considera que esta variable debe ser analizada de forma independiente en futuros estudios para determinar su naturaleza.

\section{Recomendaciones}

En una época tan relevante para la historia de Perú, se resalta la importancia de observar las fallas y los aciertos que se han tenido en materia económica y sus repercusiones sociales. El presente trabajo de investigación visibiliza la importancia que ha tenido la apertura comercial para lograr minimizar la desigualdad durante estos últimos 20 años, por lo cual políticas comerciales inclusivas y de desarrollo pueden tener un efecto en el incremento del bienestar y de la igualdad a largo plazo. Así mismo, se debe velar por la protección del consumidor y el desarrollo de una mejor masa exportadora que sea competitiva en el mercado internacional, y que pueda movilizar una mayor cantidad de empleos. Como muestran numerosos ejemplos en el mundo, las políticas distributivas bien aplicadas no son solo una afección al crecimiento económico, sino un apoyo, pues la desigualdad actúa como barrera para crecer en períodos prolongados. 


\section{Referencias bibliográficas}

Arias, J. (2020). Técnicas e instrumentos de investigación científica. Enfoques Consulting EIRL. http://hdl.handle.net/20.500.12390/223

Banco Central de Reserva del Perú (BCRP) (s.f.). Glosario de términos económicos. Obtenido de https://www.bcrp.gob.pe/docs/Publicaciones/Glosario/Glosario-BCRP.pdf

Barro, R. (1999). Inequality and Growth in a Panel of Countries. Harvard University. Obtenido de https://scholar.harvard.edu/barro/files/p_inequalitygrw.pdf

Bera A., Jarque C. (1981). Efficient tests for normality, heteroskedasticity and serial independence of regression residuals: Monte Carlo evidence. Economics Letter, 7, 313318.

Boisier, S. (2002). Conversaciones Sociales y Desarrollo Regional. Universidad de Talca.

Breusch, T.S. and A.R. Pagan, (1979), A simple test for heteroscedasticity and random coefficient variation, Econometrica, 47, 1287-1294.

Castillo, L. (2019). Patrones de la desigualdad a nivel regional en el Perú: 2007-2017. Moneda, 180(3), 13-19. https://www.bcrp.gob.pe/docs/Publicaciones/Revista-Moneda/moneda-180/moneda-180-03.pdf

Comisión Económica para América Latina y el Caribe (Cepal). La matriz de la desigualdad social en América Latina, 2016 (LC/G.2690(MDS.1/2). Obtenido de https:// repositorio.cepal.org/bitstream/handle/11362/40668/4/S1600946_es.pdf

Comisión Económica para América Latina y el Caribe (Cepal). Panorama Social de América Latina, 2019 (LC/PUB.2019/22-P/Re v.1). Obtenido de https://repositorio.cepal.org/bitstream/handle/11362/44969/5/S1901133_es.pdf

Comisión Económica para América Latina y el Caribe. (Cepal). La región ha subestimado la igualdad [Comunicado de prensa]. Obtenido de https://www.cepal.org/es/ comunicados/cepal-la-region-ha-subestimado-la-desigualdad

De Hoyos, R., \& Lustig, N. (2009). Apertura comercial, desigualdad y pobreza. El Trimestre Económico, 76(2), 283-328. https://search.proquest.com/scholarly-journals/apertura-comercial-desigualdad-y-pobreza/docview/220861834/se-2?accountid=43847

Dickey, D. A., \& Fuller, W. A. (1979). Distribution of the estimators for autoregressive time series with a unit root. Journal of the American statistical association, 74(366a), 427-431.

Durbin, J., \& Watson, G. S. (1950). Testing for serial correlation in least squares regression: I. Biometrika, 37(3/4), 409-428.

Esteban, N. (s.f.). Tipos de investigación. Obtenido de http://repositorio.usdg.edu.pe/bitstream/USDG/34/1/Tipos-de-Investigacion.pdf

Ezcurra, R., \& Rodríguez-Pose, A. (2014). Trade openness and spatial inequality in emerging Countries. Spatial Economic Analysis, 9(2), 162. https://search.proquest. $\mathrm{com} / \mathrm{scholarly-journals/trade-openness-spatial-inequality-emerging/docview/}$ $1526271431 /$ se- 2 ?accountid $=43847$

Francke, P. (2017). Una nota sobre la desigualdad de ingresos en el Perú: Mediciones recientes y preguntas pendientes. Economía, 40(79), 219-226. https://revistas.pucp. edu.pe/index.php/economia/article/view/19278 
Forbes, K. (2000). A Reassessment of the Relationship Between Inequality and Growth. American Economic Review, 90(4), 869-887. DOI: 10.1257/aer.90.4.869

Fondo Monetario Internacional. (2007). World Economic Outlook. International Monetary Fund.

Garduño, R. (2014). La apertura comercial y su efecto en la distribución regional de México. El trimestre económico, 81(322), 413-439. http://www.scielo.org.mx/scielo. php?script=sci_arttext\&pid=S2448-718X2014000200413\&lng=es\&tlng=es

Germar, V. \& Escobedo, J. (2009). Has Trade Liberalization Increased Economic Inequality among the Mexican States? An Analysis from a Spatial Econometric Perspective Economía mexicana. Nueva época, 20(1), 37-77. http://www.scielo.org.mx/scielo. php?script=sci_arttext\&pid=S1665-20452011000100002\&lng=es\&tlng=es

Glejser, H. (1969). A new test for heteroskedasticity. Journal of the American Statistical Association, 64(325), 316-323.

Granger, C. W. (1969). Investigating causal relations by econometric models and crossspectral methods. Econometrica: Journal of the Econometric Society, 37(3), 424-438. https://doi.org/10.2307/1912791

Godfrey, L.G., (1978). Testing for multiplicative heteroscedasticity, Journal of Econometrics, 8(2), 227-236. https://doi.org/10.1016/0304-4076(78)90031-3

Gómez, T., Ríos, H., \& Aali, A. (2019). Desarrollo financiero y desigualdad del ingreso, el caso de América Latina. Contaduría y Administración, 64(4), 1-17. https://doi. org/10.22201/fca.24488410e.2019.1902

Gujarati, D \& Porter, D. (2010). Heteroscedasticidad: ¿qué pasa si la varianza del error no es constante? En Econometría (pp. 379-389). México: Mc Graw Hill.

Guzmán, J. (2014). ¿La apertura comercial ha promovido el desarrollo de la innovación y la tecnología en el sector manufacturero del Perú? Instituto Nacional de Estadística e Informática. Obtenido de https://www.inei.gob.pe/media/MenuRecursivo/investigaciones/apertura-comercial-para-la-pag-web.pdf

Hernández, R., Fernández, C. \& Baptista, M. (2000). Metodología de la investigación. México: Mc Graw Hill.

Instituto Nacional de Estadística e Informática (INEI). Evolución de la pobreza monetaria 2007-2017: Informe técnico, Lima, 2018. Obtenido de https:/www.inei.gob. pe/media/cifras_de_pobreza/informe_tecnico_pobreza_monetaria_2007-2017.pdf

Ismael, P. (2007). Propuesta para la medición del desarrollo económico salarial: aplicación en doce de las principales áreas urbanas de México, 1988-2002. Papeles de población. Obtenido de http://www.scielo.org.mx/pdf/pp/v13n52/v13n52a6.pdf

López Montes, K. Benjamín, B. F., \& Alejandro, M. L. (2020). Efectos de la apertura comercial sobre la demanda de trabajo en el sector manufacturero en México. Cuadernos de Economía, 39(79), 329-354. https://doi.org/10.15446/cuad.econ.v39n79.67861

Lissardy, G. (6 de febrero de 2020). Por qué América Latina es "la región más desigual del planeta”. BBC News Mundo. Obtenido de https:/www.bbc.com/mundo/noticias-america-latina-51390621

Larios, J. \& Álvarez, V. (2014). Análisis econométrico de series de tiempo: teoría y problemas. Universidad San Ignacio de Loyola. 
Litchfield, J. (1999). Inequality: Methods and Tools. The World Bank. Obtenido de http:// icm.clsbe.lisboa.ucp.pt/docentes/url/analeco2/Papers\%5cLitchfield_Inequality_Methods_Tools.pdf

Castro-Lugo, D., \& Aguilera-Fernández, A. (2017). Apertura comercial y desigualdad salarial en México: un análisis regional para los años 1992 y 2014. Semestre Económico, 20(45), 109-131. DOI: $10.22395 /$ seec.v20n45a4

Lustig, N. (2015). La mayor desigualdad del mundo. Finanzas \& Desarrollo, 14-16. Obtenido de https://www.imf.org/external/pubs/ft/fandd/spa/2015/09/pdf/lustig.pdf

Majeed, M. (2010). Inequality, Trade Openness and Economic Growth in Asia. Applied Econometrics and International Development, 10(2). Obtenido de https://www.usc.es/ economet/reviews/aeid10216.pdf

Medina, F. (2011). Consideraciones sobre el indice de Gini para medir la concentración del ingreso. Naciones Unidas. https://repositorio.cepal.org/bitstream/handle/11362/4788/ S01020119_es.pdf?...1

Molina, O. \& Bobka, S. (2016) International trade and unexplained gender wage gaps: Evidence from agricultural sector in Bolivia. Investigación \& Desarrollo, 2(16), 4567. http://dx.doi.org/10.23881/idupbo.016.2-4e

Morán, D. (2015). Desigualdad y apertura comercial: un análisis econométrico para la economía ecuatoriana. Revista Retos, 10(2), 163-175. DOI: 10.17163/ret. n10.2015.04

Naciones Unidas (2019). Situación y perspectivas de la economía mundial en 2019: Resumen ejecutivo. Obtenido de https://www.un.org/development/desa/dpad/wp-content/uploads/sites/45/publication/WESP2019_BOOK-ES-sp.pdf

Navarro, I. (2005). Capital humano: su definición y alcances en el desarrollo local y regional. Archivos Analíticos de Políticas Educativas, 13(35), 1-36. Obtenido de https:// www.redalyc.org/pdf/2750/275020513035.pdf

Oros, L. (2015). Análisis comparativo del modelo Heckscher-Olhin y la Teoría de Linder. Tiempo económico, 29(10), 49-66. http://tiempoeconomico.azc.uam.mx/wp-content/uploads/2017/08/29te4.pdf

Piol, R. (2018). Validación de la regresión mediante el análisis de homocedasticidad. Obtenido de http://upav-biblioteca.org/site/wp-content/uploads/2018/02/Validacion-de-la-Regresion-Mediante-el-analisis-de-Homocedasticidad.pdf

Real Academia Española. (s.f.). Comercio. En Diccionario de la lengua española. Obtenido de https://dpej.rae.es/lema/comercio\#:- :texto=1.,o\%20permutando\%20g\%C3\%A9neros\%20o\%20mercanc\%C3\%ADas.

Rivera, M. T. G., \& Yserte, R. G. (2006). Disparidades económicas territoriales en el perú: Una aproximación empírica a partir de microdatos/Territorial economic disparities in peru: An empirical approach using micro data. Investigaciones Regionales, (9), 47-72. Retrieved from https://www.proquest.com/scholarly-journals/disparidades-económicas-territoriales-en-el-perú/docview/1459712243/se-2

Salas Casasola, I., Boucher, F., \& Requier-Desjardins, D. (2006). Agroindustria rural y liberalización comercial agrícola: el rol de los sistemas agroalimentarios localizados. Agroalimentaria, 11(22), 29-40. Obtenido de http://ve.scielo.org/scielo.php?scrip- 
$\mathrm{t}=\mathrm{sci}$ arttext\&pid=S1316-03542006000100003\&lng=es\&tlng=es.

Sánchez-Torres, R. (2017). Desigualdad del ingreso en Colombia: un estudio por departamentos. Cuadernos de Economía, 36(72), 139-178. http://dx.doi.org/10.15446/ cuad.econ.v36n72.65880

Sen, A. (1998). Capital humano y capacidad humana. Cuadernos de Economía, 17(29), 67-72. https://dialnet.unirioja.es/servlet/articulo? codigo $=4934956$

Sousa, V., Driessnack, M \& Costa, I. (2001). Revisión de diseños de investigación resaltantes para enfermería. Parte 1: Diseños de investigación cuantitativa. Revista Latino-Americana de Enfermagem, 15(3). https://www.scielo.br/pdf/rlae/v15n3/ es_v15n3a22.pdf

Valenzuela, J. (2008). El crecimiento económico: concepto, determinantes inmediatos y evidencia empírica. Aportes, 1(38-39), 5-32.

White, H. (1980). A heteroskedasticity-consistent covariance matrix estimator and a direct test for heteroskedasticity. Econometrica: Journal of the Econometric Society, 48(4), 817-838.

Winters, L. A., McCulloch, N., \& McKay, A. (2004). Trade liberalization and poverty: the evidence so far. Journal of economic literature, 42(1), 72-115. DOI: $10.1257 / 002205104773558056$

Wood, A. (2002). Globalization and wage inequalities: A synthesis of three theories. Weltwirtschaftliches Archiv, 138(1), 54-82. https://doi.org/10.1007/BF02707323

Wood, A. (1997). Openness and wage inequality in developing countries: the Latin American challenge to East Asian conventional wisdom. The World Bank Economic Review, 11(1), 33-57. https://doi.org/10.1093/wber/11.1.33

Zanzzi, P.; Fernández, J. \& Gonzáles, V. (2018). Relación entre la desigualdad en el ingreso, el crecimiento económico, la educación y la pobreza: una explicación desde Kuznets para Chile. Revista Espacios, 39(44), 2. http://www.revistaespacios.com/ a18v39n44/a18v39n44p31.pdf 


\section{Anexos}

Anexo 1: Prueba de Dickey-Fuller aumentada y Phillips Perron

\begin{tabular}{|c|c|c|c|c|c|c|}
\hline Serie & Test & $\begin{array}{l}\text { Modelo } \\
\text { Auxiliar }\end{array}$ & $\mathrm{T}$ - estadístico & $\begin{array}{c}\text { Prob. } \\
\text { ( } \mathrm{t} \text { - estadístico) }\end{array}$ & Estado & Conclusión \\
\hline LGINI & Phillips-Perron & $\begin{array}{l}\text { Ni tendencia } \\
\text { ni constante }\end{array}$ & -4.132763 & 0.0003 & $L G I N I-I(0)$ & $\begin{array}{l}\text { LGINI es } \mathrm{I}(0) \text {, es decir, } \\
\text { una serie estacionaria } \\
\text { en su nivel. }\end{array}$ \\
\hline$\triangle L A P E R$ & $\begin{array}{l}\text { Dickey -Fuller } \\
\text { Aumentada }\end{array}$ & $\begin{array}{l}\text { Constante y } \\
\text { tendencia }\end{array}$ & -3.787148 & 0.0382 & $\triangle L A P E R-I(0)$ & $\begin{array}{l}\triangle L A P E R \text { es } \mathrm{I}(0) \text {, es } \\
\text { decir, una serie esta- } \\
\text { cionaria en su primera } \\
\text { diferencia. }\end{array}$ \\
\hline$\triangle L C R E D$ & $\begin{array}{l}\text { Dickey -Fuller } \\
\text { Aumentada }\end{array}$ & $\begin{array}{l}\text { Ni tendencia } \\
\text { ni constante }\end{array}$ & -2.488627 & 0.0156 & $\triangle L C R E D \sim I(0)$ & $\begin{array}{l}\triangle L C R E D \text { es } \mathrm{I}(0) \text {, es } \\
\text { decir, una serie esta- } \\
\text { cionaria en su primera } \\
\text { diferencia. }\end{array}$ \\
\hline$\triangle L P O P S H$ & $\begin{array}{l}\text { Dickey -Fuller } \\
\text { Aumentada }\end{array}$ & $\begin{array}{l}\text { Constante y } \\
\text { tendencia }\end{array}$ & 5.273890 & 0.031 & $L C H-I(0)$ & $\begin{array}{l}\triangle L P O P S H \text { es } \mathrm{I}(0) \text {, es } \\
\text { decir, una serie esta- } \\
\text { cionaria en su primera } \\
\text { diferencia. }\end{array}$ \\
\hline$\triangle L C A P H U M$ & $\begin{array}{l}\text { Dickey -Fuller } \\
\text { Aumentada }\end{array}$ & $\begin{array}{l}\text { Ni tendencia } \\
\text { ni constante }\end{array}$ & -2.067756 & 0.0397 & $\triangle L C A P-I(0)$ & $\begin{array}{l}\triangle L C A P H U M \text { es } \mathrm{I}(0) \text {, } \\
\text { es decir, una serie esta- } \\
\text { cionaria en su primera } \\
\text { diferencia. }\end{array}$ \\
\hline$\triangle L A G R$ & $\begin{array}{l}\text { Dickey -Fuller } \\
\text { Aumentada }\end{array}$ & Constante & -4.363619 & 0.0029 & $\triangle L A G R \sim I(0)$ & $\begin{array}{l}\triangle L A G R \text { es I }(0) \text {, es decir, } \\
\text { una serie estacionaria } \\
\text { en su primera diferen- } \\
\text { cia. }\end{array}$ \\
\hline$\triangle L I N D$ & $\begin{array}{l}\text { Dickey -Fuller } \\
\text { Aumentada }\end{array}$ & Constante & -4.688645 & 0.0064 & $\triangle L I N D \sim I(0)$ & $\begin{array}{l}\triangle L I N D \text { es } \mathrm{I}(0) \text {, es decir, } \\
\text { una serie estacionaria } \\
\text { en su primera diferen- } \\
\text { cia. }\end{array}$ \\
\hline
\end{tabular}


Anexo 2: Prueba de causalidad de Granger

\begin{tabular}{|c|c|c|c|c|c|}
\hline Relación de causalidad & F-estadístico & $\begin{array}{l}\text { Probabilidad } \\
\text { F-estadístico }\end{array}$ & $\begin{array}{c}\text { Criterio de } \\
\text { información }\end{array}$ & $\begin{array}{c}\text { Orden de } \\
\text { Rezago }\end{array}$ & Observaciones \\
\hline$\triangle L A P E R$ granger $\rightarrow$ LGINI & 7.41522 & 0.0643 & $\mathrm{SIC}$ & 6 & 16 \\
\hline LGINI granger $\rightarrow \triangle A P E R$ & 3.49226 & 0.0780 & $\mathrm{SIC}$ & 1 & 21 \\
\hline$\triangle C R E D$ granger $\rightarrow$ LGINI & 2.68457 & 0.1008 & $\mathrm{SIC}$ & 2 & 20 \\
\hline LGINI granger $\rightarrow \triangle L P O P S H$ & 5.82691 & 0.0882 & $\mathrm{SIC}$ & 6 & 16 \\
\hline$\triangle L C A P H U M$ granger $\rightarrow$ LGINI & 7.09630 & 0.0073 & $\mathrm{SIC}$ & 4 & 18 \\
\hline LGINI granger $\rightarrow \triangle L C A P H U M$ & 5.30184 & 0.0330 & $\mathrm{SIC}$ & 5 & 17 \\
\hline$L A G R$ granger $\rightarrow$ LGINI & 4.01164 & 0.0388 & $\mathrm{SIC}$ & 4 & 18 \\
\hline DLIND granger $\rightarrow$ LGINI & 9.34927 & 0.0470 & $\mathrm{SIC}$ & 6 & 16 \\
\hline LGINI granger $\rightarrow$ DLIND & 5.25145 & 0.1008 & $\mathrm{SIC}$ & 6 & 16 \\
\hline$\triangle L A P E R$ granger $\rightarrow \triangle L A G R$ & 4.29665 & 0.0335 & $\mathrm{SIC}$ & 2 & 20 \\
\hline$\triangle L A G R$ granger $\rightarrow \triangle L A P E R$ & 5.75859 & 0.0140 & SIC & 4 & 18 \\
\hline$\triangle L C R E D$ granger $\rightarrow \triangle L A P E R$ & 6.38698 & 0.0211 & $\mathrm{SIC}$ & 1 & 21 \\
\hline$\triangle L C R E D$ granger $\rightarrow \triangle L C A P H U M$ & 15.2755 & 0.0238 & $\mathrm{SIC}$ & 6 & 16 \\
\hline$\triangle L C A P H U M$ granger $\rightarrow \triangle L C R E D$ & 3.89476 & 0.0640 & $\mathrm{SIC}$ & 1 & 21 \\
\hline$\triangle L A G R$ granger $\rightarrow \triangle L C R E D$ & 6.63388 & 0.0086 & $\mathrm{SIC}$ & 2 & 20 \\
\hline$\triangle L P O P S H$ granger $\rightarrow \triangle L A G R$ & 3.39283 & 0.0845 & SIC & 5 & 17 \\
\hline$\triangle L P O P S H$ granger $\rightarrow \triangle L C A P H U M$ & 4.56722 & 0.0459 & $\mathrm{SIC}$ & 5 & 17 \\
\hline$\triangle L C A P H U M$ granger $\rightarrow \triangle L A G R$ & 4.12242 & 0.0374 & SIC & 2 & 20 \\
\hline$\triangle L C A P H U M$ granger $\rightarrow \triangle L A P E R$ & 14.9182 & 0.0246 & $\mathrm{SIC}$ & 6 & 16 \\
\hline$\triangle L C A P H U M$ granger $\rightarrow \triangle L A G R$ & 4.12242 & 0.0374 & $\mathrm{SIC}$ & 2 & 20 \\
\hline$\triangle L I N D$ granger $\rightarrow \triangle L C A P H U M$ & 6.29775 & 0.0103 & $\mathrm{SIC}$ & 2 & 20 \\
\hline
\end{tabular}




\section{Anexo 3: MCO MODELO GRANGER}

\begin{tabular}{|c|c|c|c|c|}
\hline \multicolumn{5}{|c|}{ Dependent Variable: LGINI } \\
\hline \multicolumn{5}{|l|}{ Method: Least Squares } \\
\hline \multicolumn{5}{|c|}{ Included observations: 22 after adjustments } \\
\hline Variable & Coefficient & Std. Error & t-Statistic & Prob. \\
\hline C & 3.814848 & 0.033254 & 114.7201 & 0.0000 \\
\hline DLAPERTURA & 0.230910 & 0.273250 & 0.845050 & 0.4105 \\
\hline DLCREDITO & -0.017952 & 0.242568 & -0.074007 & 0.9419 \\
\hline DLCAPHUMANO & 5.432075 & 2.850183 & 1.905869 & 0.0748 \\
\hline DLAGRICOLA & -0.678042 & 1.300837 & -0.521235 & 0.6093 \\
\hline DLINDUSTRIAL & 0.129059 & 1.103640 & 0.116939 & 0.9084 \\
\hline R-squared & 0.269041 & \multicolumn{2}{|c|}{ Mean dependent var } & 3.859632 \\
\hline Adjusted R-squared & 0.040617 & \multicolumn{2}{|c|}{ S.D. dependent var } & 0.089813 \\
\hline S.E. of regression & 0.087971 & \multicolumn{2}{|c|}{ Akaike info criterion } & -1.796629 \\
\hline Sum squared resid & 0.123821 & \multicolumn{2}{|c|}{ Schwarz criterion } & -1.499072 \\
\hline Log likelihood & 25.76292 & \multicolumn{2}{|c|}{ Hannan-Quinn criter. } & -1.726534 \\
\hline F-statistic & 1.177813 & \multicolumn{2}{|c|}{ Durbin-Watson stat } & 0.595402 \\
\hline Prob(F-statistic) & 0.362737 & & & \\
\hline
\end{tabular}

\section{Anexo 4: MCO MODELO CORREGIDO}

\begin{tabular}{|c|c|c|c|c|}
\hline \multicolumn{5}{|c|}{ Dependent Variable: LGINI } \\
\hline \multicolumn{5}{|l|}{ Method: Least Squares } \\
\hline \multicolumn{5}{|c|}{ Included observations: 22 after adjustments } \\
\hline Variable & Coefficient & Std. Error & $\mathrm{t}$-Statistic & Prob. \\
\hline c & 3.919244 & 0.015764 & 248.6213 & 0.0000 \\
\hline DLAPERTURA & -0.303810 & 0.173681 & -1.749239 & 0.1007 \\
\hline DLCAPHUMANO & 3.369798 & 0.916304 & 3.677597 & 0.0022 \\
\hline D2011DLCAPHUMANO & -27.74695 & 3.104159 & -8.938637 & 0.0000 \\
\hline D200901 & -0.104875 & 0.058492 & -1.792995 & 0.0932 \\
\hline D201101 & 0.058851 & 0.037541 & 1.567622 & 0.1378 \\
\hline D2019 & -0.051139 & 0.033473 & -1.527756 & 0.1474 \\
\hline R-squared & 0.913945 & \multicolumn{2}{|c|}{ Mean dependent var } & 3.859632 \\
\hline Adjusted R-squared & 0.879523 & \multicolumn{2}{|c|}{ S.D. dependent var } & 0.089813 \\
\hline S.E. of regression & 0.031174 & \multicolumn{2}{|c|}{ Akaike info criterion } & -3.845086 \\
\hline Sum squared resid & 0.014577 & \multicolumn{2}{|c|}{ Schwarz criterion } & -3.497937 \\
\hline Log likelihood & 49.29595 & \multicolumn{2}{|c|}{ Hannan-Quinn criter. } & -3.763308 \\
\hline F-statistic & 26.55108 & \multicolumn{2}{|c|}{ Durbin-Watson stat } & 1.834956 \\
\hline Prob(F-statistic) & 0.000000 & & & \\
\hline
\end{tabular}

